\title{
Embedded inertial measurement unit reveals pole lean angle for cross-country skiing
}

\author{
John Bruzzo ${ }^{1}$ (D) Noel C. Perkins ${ }^{2} \cdot$ Aki Mikkola $^{1}$
}

Published online: 3 March 2020

(c) The Author(s) 2020

\begin{abstract}
This study introduces an inertial measurement unit-based measurement system for resolving the dynamic lean angle of a ski pole during double poling while cross-country skiing. The measurement system estimates both the pole lean angle and pole-terrain contact events. Reported are results from 20 trials providing validated estimates of ski pole lean angle and the timing of pole plant and pole lift events. The pole lean angle is estimated from a complementary filter that fuses estimates of orientation from the embedded accelerometer and angular rate gyro. Validation follows from comparison with video capture measurements. Bland-Altman analysis showed agreement between the two measurement modalities with less than $5 \%$ bias in the mean differences (relative to the lean angle range of motion). Companion correlation analysis confirms strong correlation $(r=0.99)$ between the inertial measurement unit and video-estimated lean angles and with mean root-mean-square errors below $4.5^{\circ}$.
\end{abstract}

Keywords Cross-country skiing $\cdot$ Ski pole $\cdot$ Inertial measurement unit $\cdot$ Ski pole kinematics

\section{Introduction}

Poling serves as a major source of propulsion in the classic (diagonal-stride) style of cross-country skiing and becomes the main source when double poling (poling synchronously with both arms) is employed [1]. The pole forces developed during double poling (DP) and the optimum timing to maximize those forces remain of keen interest to coaches and athletes, as they seek to increase poling power and hence skiing speed. This interest spans the entire range of skiing events from sprint courses to long-distance marathons where DP remains prevalent [2]. Prior studies highlight the overall importance and influence of the poling forces arising from DP [1, 3-12].

This article is a part of Topical Collection in Sports Engineering on Winter Sports, edited by Dr. Aimee Mears, Dr. David Pearsall, Dr. Irving Scher and Dr. Carolyn Steele.

John Bruzzo

john.bruzzo@lut.fi

1 Laboratory of Machine Design, Lappeenranta University of Technology, 53850 Lappeenranta, Finland

2 Department of Mechanical Engineering, University of Michigan, Ann-Arbor, MI 48109, USA
Simultaneous knowledge of (1) the total force applied by the pole to the terrain, (2) the angle of the pole with respect to the terrain, and (3) the timing of pole-terrain contact enables one to deduce the component of the pole force applied parallel to the terrain, the time interval over which it is applied, and consequently the forward impulse responsible for propulsion. The ability to measure these three factors while actively skiing outdoors on a course remains a clear challenge. These studies [1,3-12] have measured these factors only in laboratory settings or in highly controlled conditions that preclude characterizing propulsion on outdoor courses.

To succeed in studying propulsion on outdoor courses, one could combine pole-embedded force transducers with inertial measurement units (IMUs) to estimate pole orientation with respect to the terrain. Doing so would provide the essential information to estimate the component of the poling force in the plane of the terrain that is responsible for propulsion (rather than weight bearing). However, simply measuring the pole orientation, such as the pole lean angle with respect to the vertical, remains a challenge in outdoor skiing because of the lack of portability of the measurement devices for this task, their high sensitivity to weather conditions, and the high number of accessories needed for their correct operation. Camera-based systems for measuring 
pole orientation based on video $[8,13-15]$ or infrared $[1,5$, $10-12,16,17]$ motion capture are ill suited for the purpose of outdoor testing and over the large distances required. A potential solution proposed in this study is a pole-embedded IMU to estimate the dynamic pole lean angle. Moreover, IMUs represent an attractive solution due to their low cost and miniaturization.

While IMUs have been deployed to study skiing technique [18-21], they have not been used to study the motion of the pole beyond detecting pole-terrain contact events, cycle duration, cycle speed, and cycle length [19, 22]. Nevertheless, a pole-embedded IMU also provides the requisite data (acceleration and angular velocity) needed to estimate the lean angle of the pole as well. Thus, the objective of this study was to introduce an IMU-based system to estimate the dynamic pole lean angle of skiers performing the DP technique and, importantly, to determine the accuracy of the estimated pole lean angles by comparison with those measured using video recordings.

\section{Methods}

A skilled skier, with experience in cross-country skiing competitions in the range of $30 \mathrm{~km}$ in national racing, acted as the test participant following requirements of The Finnish Advisory Board for Research Integrity. Data collecting and handling complied with the requirements of the Finnish Data Protection Ombudsman. The participant provided informed consent for the study.

\subsection{Experimental facility and protocol}

The test facility was a flat and level outdoor ski track equipped for video recording within a short $(9 \mathrm{~m})$ field of view; refer to schematic of ski track (top view) in Fig. 1. At the beginning of a trial, the participant warmed up according to individual routine. The participant then performed ten trials utilizing the DP technique with self-selected skiing speeds using classic-style skis. Following a 3-minute break, the participant performed an additional 10 trials (total 20 trials). The field of view of the video camera was sufficient to resolve two poling cycles per trial yielding a total of 40 poling cycles for the study. An embedded IMU provided simultaneous data (linear acceleration and angular velocity) sampled from the pole as described next.

\subsection{Selection of the skiing technique}

The DP technique induces two-dimensional motion of the pole (motion largely in the saggital plane) as seen in previous studies $[1,27]$. This motion can be measured using a

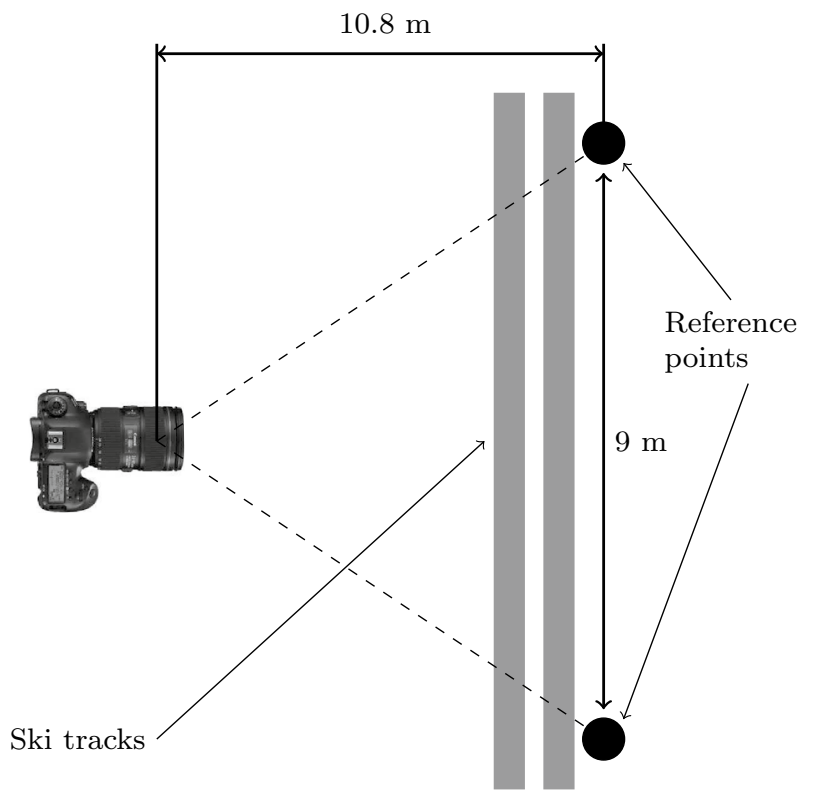

Fig. 1 Experimental facility. Schematic (top view) of ski track with location of camera and field of view

camera system with its optical axes aligned perpendicular to this plane.

\subsection{Equipment specifications and data analysis}

The study employed a ski pole (1.450 m length, R2 Competition Pole AH03-4R2S145, Rex, Finland) that was modified with two markers to support video imaging and custom instrumentation to support inertial motion sensing; refer to Fig. 2a.

For video imaging, one (top) marker was attached near the handgrip ( $250 \mathrm{~mm}$ below the top of the pole) and a second (bottom) marker was attached near the tip of the pole (300 $\mathrm{mm}$ above the tip) (Fig. 2a). A stationary camera (Cannon EOS 700, Japan), having a $50 \mathrm{~Hz}$ frame rate, filmed the motion of the pole (Fig. 1). The $(x, y)$ coordinates of the pole-attached markers were extracted automatically from the video recordings using the open-source program Tracker 4.11.0 (Open Source Physics, USA) with a position measurement resolution of $1 \mathrm{~mm}$. Given the known distance between the two markers $(950 \mathrm{~mm})$, the expected resolution of the lean angle measured in the vertical plane from the video images is $\arctan (2 / 950)=0.12^{\circ}$. The procedure utilized here was similar to that described in [19].

The custom instrumentation for inertial motion sensing consisted of a six-degree of freedom IMU (MPU-6050, InvenSense, USA) capable of resolving both acceleration (acceleration range of $\pm 16 \mathrm{~g}$ and a noise floor of 400 $\mu \mathrm{g} / \sqrt{\mathrm{Hz}}$ ) and angular velocity (angular velocity range of $\pm 2000 \%$ s and a noise floor of $0.005 \% / \mathrm{s} / \sqrt{\mathrm{Hz}}$ ), and an 


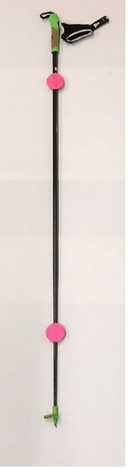

(a)

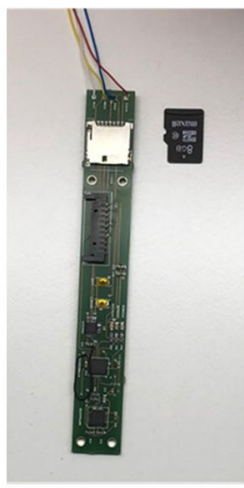

(b)

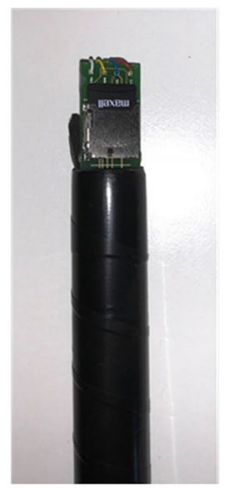

(c)
Fig. 2 a Ski pole used in study with top and bottom markers and pole-embedded instrumentation. b Major components of the instrumentation mounted to a custom, slender pc board includes a six degree-of-freedom IMU (MPU-6050, Invensense, USA), amplifier (INA133, Texas Instruments, USA), microcontroller (ATMEGA328 Microchip, USA), a micro SD card (8 GB MicroSDHC, Maxell, UK), and battery (9 V/6LP3146, Duracell, Malaysia. Not shown). c Instrumentation inserted in the hollow of the handgrip. A cap (not shown) on the handgrip seals the instrumentation cavity

associated data acquisition (DAC) system (prototype, LUT, Finland), both mounted to a miniature pc board measuring $13 \mathrm{~mm} \times 100 \mathrm{~mm}$ (Fig. 2b). This slender design enabled the IMU and DAC system to fit within the hollow of the handgrip and with an overall added mass of $53 \mathrm{~g}$ (DAC board: 8 g/Battery: $45 \mathrm{~g}$ ). The DAC sampled the acceleration and the angular velocity at a rate of $300 \mathrm{~Hz}$ and wrote the data to a 8 $\mathrm{Gb}$ micro SD card. This design enabled continuous sampling over $6 \mathrm{~h}$. Figure $2 \mathrm{~b}$ illustrates the custom instrumentation and its installation within the grip of the pole (Fig. 2c). A small cap was added to the handgrip that covers the instrumentation (not shown).

The IMU was calibrated to reduce bias error and crossaxis sensitivity errors according to the procedure detailed in [23]. The raw IMU data was filtered using a $2 \mathrm{~Hz}$ low-pass second-order Butterworth digital filter to yield smoothed data similar to the procedure in [20]. Note that the dominant (pendulum-like) motion of the pole does not possess frequencies significantly above $2 \mathrm{~Hz}$ as confirmed by the power spectrum of the acceleration data. All data processing was performed using standard MATLAB functions (The MathWorks, Natick, MA, USA).

To compare the estimates of the pole motion measured by video and IMU data, the two data streams were time synchronized. The time synchronization was achieved using the following three-stage procedure. Each test session, consisting of ten trials, was filmed in one continuous video recording. At the start of each test session, three pole "movement events" were introduced, which were readily discernible in both the video recordings and the IMU data to establish synchronization times. First, the trial started with a static pose of the pole. The static pose period is readily identifiable in the IMU data (period of sustained zero angular velocity) and the start and end of this static pose period are also clearly identifiable in the video recordings. Second, the pole was rotated $90^{\circ}$ in the vertical plane with static poses between two successive rotations. These two static poses yield additional synchronization times. Finally, the pole was rotated (at a priori unknown angular velocity) starting and ending with a static pose. Again, the resulting static poses and angular motions are readily identifiable in both the video recordings and IMU readings. Simultaneous use of the resulting three sets of synchronization times enabled minimization of the synchronization error across these times. It is assumed that this time synchronization was maintained during the length of each of the test sessions attributed to any possible inaccuracy generated from the real-time clock utilized in the custom instrumentation.

\subsection{Estimation of ski pole orientation in DP}

The orientation of the ski pole in DP is largely determined by the lean angle $\theta$ (in the sagittal plane relative to the vertical and the direction of skiing) as presented in [20].

The lean angle is calculated by fusing independent estimates of the vertical direction using the accelerometer data and the angular velocity data by using a modification of the complementary filter presented in [24]. The modified filter leads to an estimate of the "true" angular velocity over each time step by correcting for the differing estimates of vertical (direction of gravity) found from the acceleration data versus the original angular velocity data. Doing so leverages the measured linear acceleration to estimate the bias error and noise present in the angular velocity data and to then remove those error sources in arriving at the "true angular velocity" as follows.

Figure 3 illustrates a block diagram of the complementary filter that returns estimates of the lean angle at each time step following five major steps summarized as follows. In the first step, the direction of gravity at the next time step is estimated from the acceleration data assuming that the accelerometer functions as an inclinometer. The resulting direction of gravity is denoted $\mathbf{G}_{a}$. Next, the orientation of the IMU at the next time step is estimated by integrating the angular velocity forward one time step. Doing so yields a second estimate of the direction of gravity (i.e., the vertical direction) and this estimate is denoted $\mathbf{G}_{\omega}$. In the third step, the (angular) difference of the two estimates yields the misalignment denoted $\boldsymbol{\Theta}_{e}$. The misalignment is then used to correct the original angular velocity over the same time step in arriving at the "true" angular velocity for that time step denoted $\boldsymbol{\Omega}$. Finally, the corrected orientation of the IMU is found by integrating the true angular velocity forward one 
Fig. 3 Inputs, steps, and output of the complementary filter implemented in [24]

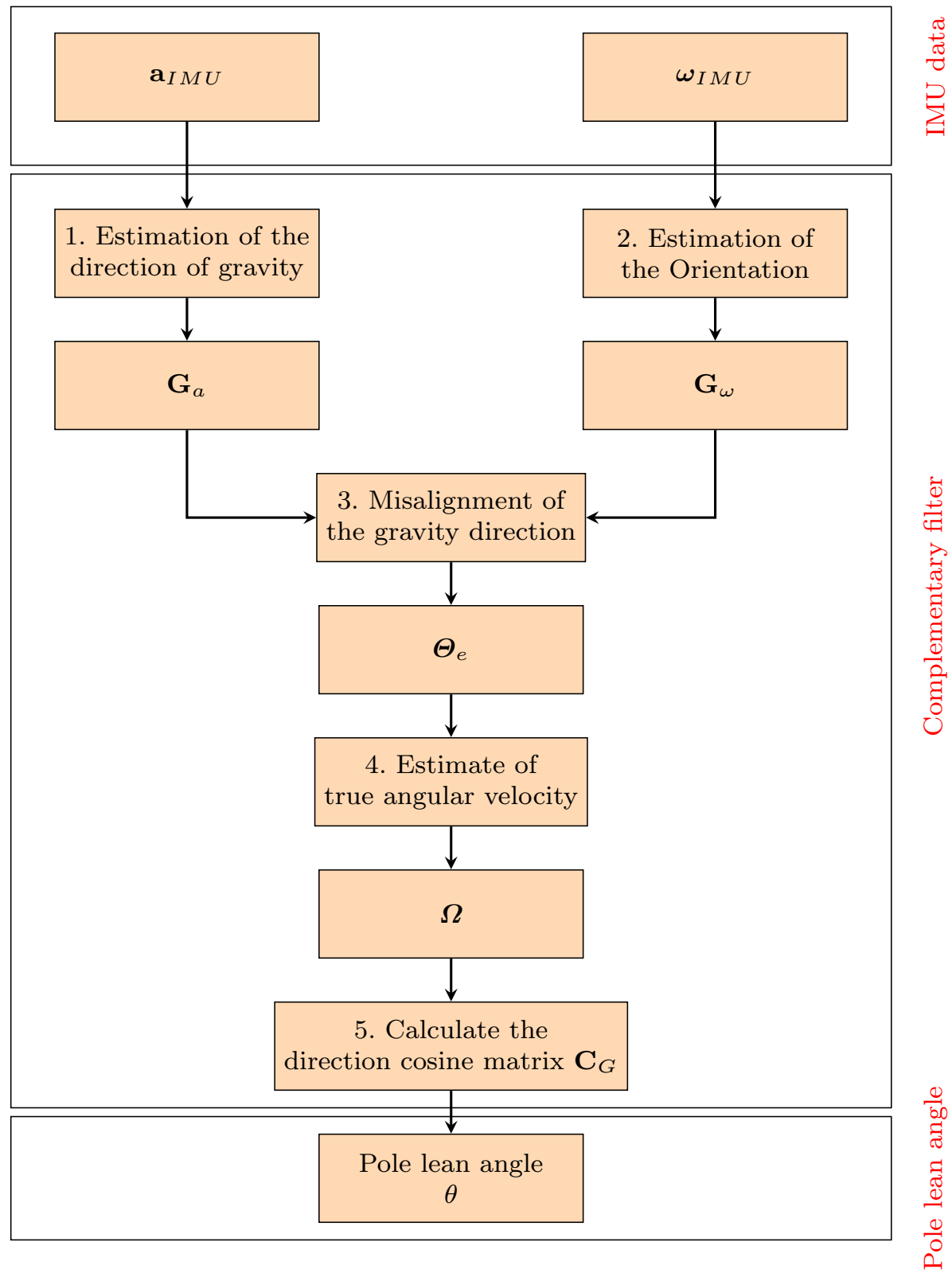

time step. Doing so yields the direction cosine matrix $\mathbf{C}_{G}$ at the new time step from which the associated lean angle is computed. This process, summarized in Fig. 3, is repeated for each time step. Refer to [24] for further details.

\subsection{Ski pole event detection}

The magnitude of the acceleration measured by the IMU embedded in the grip reveals the major phases of ski pole motion. The motion phases include the plant phase that begins the instant the pole tip is first planted on the terrain, the instant that the tip is lifted from the snow, and the follow-on swing phase that precedes the next plant. Figure 4 illustrates these pole contact events over two complete poling cycles for a

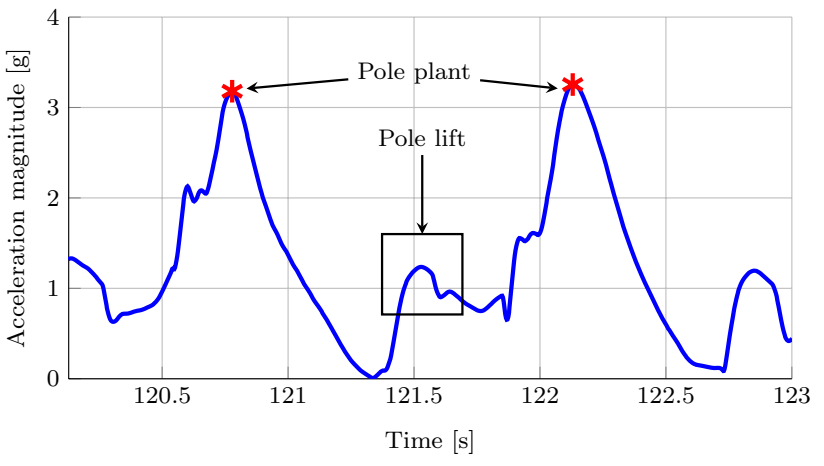

Fig. 4 Magnitude of the acceleration measured by the IMU embedded in the handgrip reveals the pole plants and lifts over two poling cycles for diagonal stride skiing 
typical trial. The instants of pole plants are readily observable in this plot of acceleration magnitude versus time, since the highest acceleration peaks arise when the pole first contacts the terrain $[25,26]$. Pole lifts arise within the lower acceleration peaks between two successive pole plants. During the plant phase, the pole is under compression and that force (directed along the axis of the pole) generates a vertical ground reaction and, importantly, also a horizontal ground reaction that propels the skier forward $[1,4,27]$. Note that while the acceleration magnitude reveals a time region $( \pm 0.13 \mathrm{~s}$ as observed in the plots) where pole lift occurs, the precise time of that lift is undetectable without further analysis.

Our proposed analysis exploits the kinematic constraint that, during the plant phase, the tip of the pole remains planted. Consequently, the acceleration measured at the grip must also depend on the angular velocity (and angular acceleration) of the pole as measured by the grip-embedded angular rate gyro. To reveal that, we introduce the three position vectors illustrated in Fig. 5 that locate the position of the tip of the pole (point T), the position of the IMU within the grip (point I), and the position of $\mathrm{T}$ relative to $\mathrm{I}$.

During the plant phase, the angular velocity of the pole, denoted $\omega$, can be used to estimate the acceleration measured at the location of embedded IMU, denoted $\mathbf{a}_{I, \text { est }}$, through

$\mathbf{a}_{(I, \mathrm{est})}=\alpha \times \mathbf{r}_{I / T}+\omega \times\left(\omega \times \mathbf{r}_{I / T}\right)$,

where $\alpha$ is the angular acceleration of the pole (obtained from $\boldsymbol{\omega}$ by central finite differencing). During the plant phase, the magnitude of $\mathbf{a}_{I, \text { est }}$ must remain within a small threshold of that measured directly by the embedded accelerometer, denoted $\mathbf{a}_{I}$, per

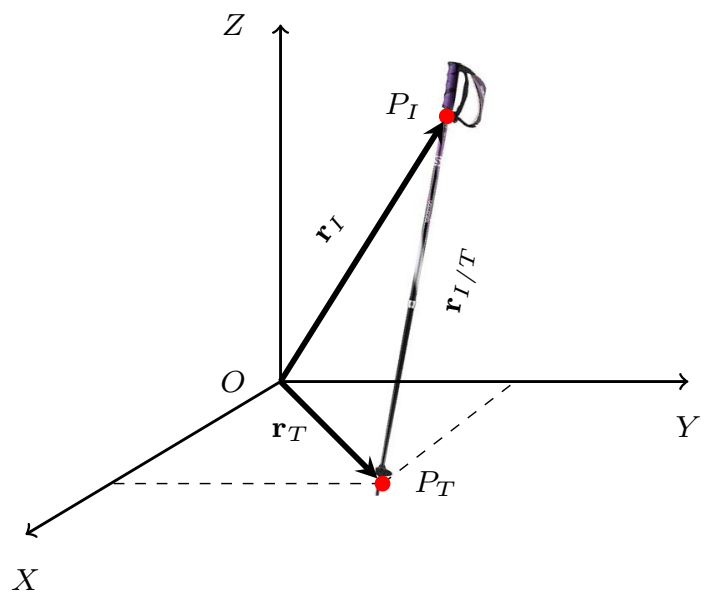

Fig. 5 Schematic illustrating the position of the tip (T) of the pole, the IMU (I) embedded in the grip, and the position of point I relative to point $\mathrm{P}$
\|\| $\mathbf{a}_{I, \text { est }}\|-\| \mathbf{a}_{I} \| \mid \leq a_{\text {threshold }}$

where the superimposed component of gravity in $\mathbf{a}_{I}$ is duly subtracted. To estimate the quantity threshold $a_{\text {threshold }}$, four poling cycles representing $10 \%$ of total cycles in the experiment were randomly selected. A video analysis of these four poling cycles showed the instants of pole lift, and from this a threshold value of $a_{\text {threshold }}=0.05 \mathrm{~g}$ succeeds in identifying the lifts.

\subsection{Assessment of agreement}

The agreement between the IMU-estimated lean angles and those measured by the video recording was assessed using Bland-Altman analysis [28]. Prior to applying the Bland-Altman analysis, a test for normality was carried out on the distribution of the differences using the Kolmogorov-Smirnov (KS) test [29]. In cases where the differences failed the normality test, a logarithmic transformation was applied. The range of motion (ROM) of the pole lean angle was used to define limits of $5 \%$ of the maximum bias for the mean difference and $10 \%$ of the maximum limits for the 95\% confidence interval. These limits determine if the bias and confidence intervals from the Bland-Altman analysis yield acceptable agreement. The measurement error for the trials was qualitatively categorized (random or systematic) according to the variability of the differences observed in the Bland-Altman plots as detailed in [30].

\section{Results and discussion}

The pole lean angle estimated using the IMU data is compared to that measured from the video images. Subsequently, the IMU-derived pole-terrain contact events are compared with those deduced from the video images.

\subsection{Ski pole lean angle}

Across all 20 trials considered in this study, the pole lean angles estimated from IMU data agree with those measured from the video images. This agreement is first observed qualitatively in Fig. 6 which compares the angles estimated by both measurement modalities for poling cycles from two sample trials. These trials, which are representative of all trials, also show variation across poling cycles. In particular, the poling cycle time and range of motion for the trial on the left (Fig. 6a) differ from those shown on the right (Fig. 6b) by $0.21 \mathrm{~s}$ and $12^{\circ}$, respectively. While the experiments were not intended to study cycle-to-cycle variations and possible differences in poling techniques, these variations are easy to observe and may well be useful in studying poling technique in future studies. 


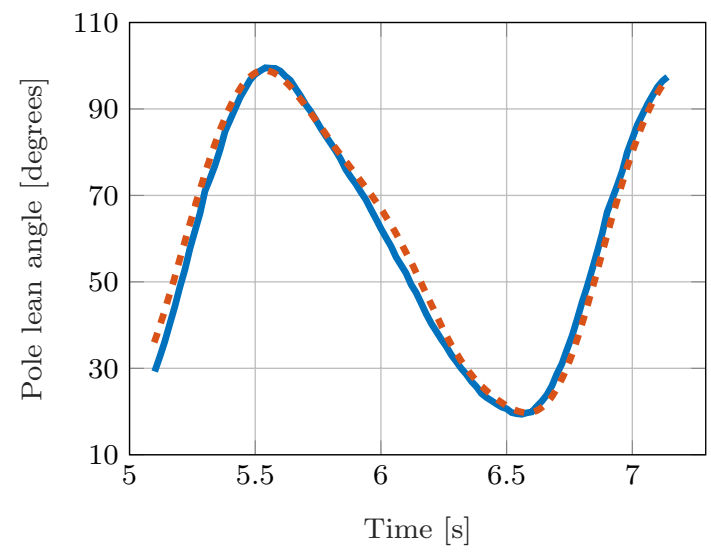

(a)

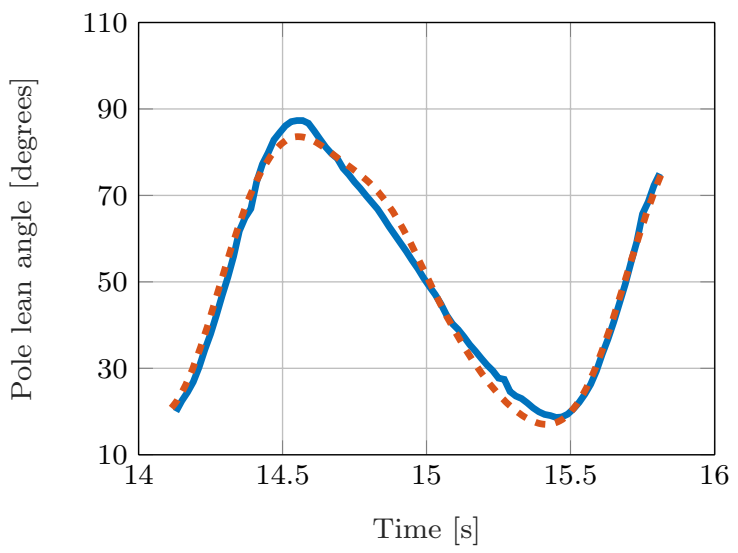

(b)

Fig. 6 Pole lean angle estimated by IMU data (dashed) and by video images (solid) are plotted versus time for two example poling cycles: a trial 4 , and $\mathbf{b}$ trial 15

Quantitative comparisons of the agreement between the two measurement modalities are confirmed from the Bland-Altman and correlation analyses reported in Tables 1 and 2, respectively, across all 20 trials. Additionally, a sample set of Bland-Altman and correlation plots for a representative trial (that appearing in Fig. 6a) are presented in Fig. 7.

Inspection of the sample Bland-Altman plot, Fig. 7a, reveals that the two measurement modalities yield highly comparable estimates of the pole lean angle. The differences in the angular measurements remain well within the

Table 1 Agreement as assessed using Bland-Altman analysis

\begin{tabular}{|c|c|c|c|c|c|c|c|c|c|}
\hline Trial & $\begin{array}{l}\text { Number of } \\
\text { points }(n)\end{array}$ & $\begin{array}{l}\text { Difference } \\
\text { mean }(\bar{d})\end{array}$ & $\begin{array}{l}\text { Standard devia- } \\
\text { tion (SD) }\end{array}$ & $\bar{d}-1.96 s$ & $\bar{d}+1.96 s$ & KS & Type & Bias error $(\%)$ & $\operatorname{ROM}\left({ }^{\circ}\right)$ \\
\hline 1 & 149 & 0.69 & 1.98 & -3.2 & 4.6 & 0.56 & Systematic & 1.08 & 64 \\
\hline 2 & 97 & -0.52 & 2.87 & -6.1 & 5.1 & 0.55 & Systematic & 0.76 & 68 \\
\hline 3 & 139 & -0.82 & 3.23 & -7.2 & 5.5 & 0.37 & Systematic & 1.19 & 69 \\
\hline 4 & 110 & 0.99 & 3.35 & -5.6 & 7.6 & 0.48 & Systematic & 1.30 & 76 \\
\hline 5 & 105 & 4.00 & 4.50 & -4.8 & 13.0 & 0.01 & Systematic & 5.71 & 70 \\
\hline 6 & 106 & -0.96 & 2.74 & -6.3 & 4.4 & 0.05 & Systematic & 0.17 & 82 \\
\hline 7 & 123 & 1.20 & 3.96 & -6.6 & 9.0 & 0.03 & Systematic & 1.41 & 85 \\
\hline 8 & 114 & -0.50 & 3.53 & -7.4 & 6.4 & 0.62 & Systematic & 0.70 & 71 \\
\hline 9 & 129 & -0.96 & 2.05 & -5.0 & 3.1 & 0.12 & Systematic & 1.52 & 63 \\
\hline 10 & 105 & -1.04 & 3.30 & -7.5 & 5.4 & 0.55 & Systematic & 1.32 & 79 \\
\hline 11 & 129 & 0.86 & 2.17 & -3.4 & 5.1 & 0.47 & Systematic & 1.18 & 73 \\
\hline 12 & 123 & -1.11 & 2.37 & -5.8 & 3.5 & 0.55 & Systematic & 1.86 & 60 \\
\hline 13 & 131 & 0.90 & 2.59 & -4.2 & 6.0 & 0.30 & Systematic & 1.43 & 63 \\
\hline 14 & 112 & -1.18 & 3.10 & -7.3 & 4.9 & 0.37 & Systematic & 1.62 & 73 \\
\hline 15 & 119 & -1.40 & 2.50 & -6.3 & 3.5 & 0.32 & Systematic & 2.46 & 57 \\
\hline 16 & 118 & 1.08 & 1.11 & -1.1 & 3.3 & 0.54 & Systematic & 1.81 & 60 \\
\hline 17 & 105 & -3.03 & 2.25 & -7.4 & 1.4 & 0.33 & Systematic & 4.27 & 71 \\
\hline 18 & 113 & 1.45 & 2.68 & -3.8 & 6.7 & 0.06 & Systematic & 2.10 & 69 \\
\hline 19 & 98 & -2.31 & 3.01 & -8.2 & 3.6 & 0.48 & Systematic & 3.30 & 70 \\
\hline 20 & 135 & -2.22 & 1.55 & -5.3 & 0.8 & 0.07 & Systematic & 3.63 & 61 \\
\hline
\end{tabular}

The table reports the number of samples $(n)$ in each trial, the difference mean $(\bar{d})$, the standard deviation (SD) of the differences, the $95 \%$ confidence interval of the agreement limits $(-1.96 \bar{d},+1.96 \bar{d})$, the $p$ values from the KS test to check for normality of the differences, the qualitative type of error observed on the scatter, the bias error, and range of motion (ROM) of the pole lean angle 


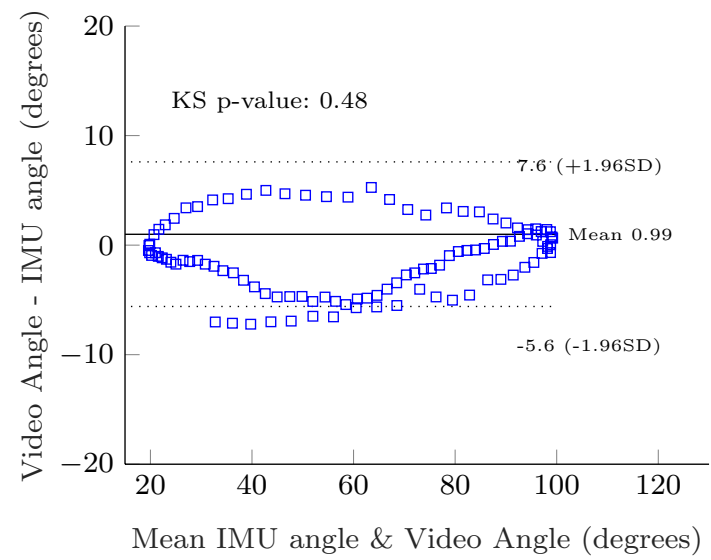

(a)

Fig. 7 Sample Bland-Altman and correlation analysis for example of Fig. 6a. a Bland-Altman plot compares the pole lean angles estimated by the IMU against those measured by video. Solid line illustrates the bias of the mean differences $(\bar{d}=0.99)$ and the dotted

Table 2 Agreement as assessed using correlation analysis

\begin{tabular}{lllllr}
\hline Trial & $\begin{array}{l}\text { Number of } \\
\text { points }(n)\end{array}$ & $\begin{array}{l}\text { Rms differ- } \\
\text { ence }\left({ }^{\circ}\right)\end{array}$ & $r$ & $m$ & $b\left(^{\circ}\right)$ \\
\hline 1 & 149 & 1.95 & 0.996 & 1.02 & -0.21 \\
2 & 97 & 2.59 & 0.994 & 1.05 & -3.71 \\
3 & 139 & 3.02 & 0.989 & 0.95 & 1.73 \\
4 & 110 & 2.35 & 0.994 & 1.05 & -1.31 \\
5 & 105 & 4.51 & 0.982 & 1.01 & 3.55 \\
6 & 106 & 2.75 & 0.995 & 1.01 & -1.33 \\
7 & 123 & 3.96 & 0.991 & 0.99 & 1.53 \\
8 & 114 & 2.73 & 0.995 & 1.09 & -6.46 \\
9 & 129 & 1.80 & 0.997 & 1.05 & -3.90 \\
10 & 105 & 3.31 & 0.993 & 1.00 & -1.32 \\
11 & 129 & 2.86 & 0.993 & 1.01 & -1.07 \\
12 & 123 & 2.16 & 0.990 & 1.06 & -4.12 \\
13 & 131 & 1.56 & 0.989 & 1.02 & -2.09 \\
14 & 112 & 3.03 & 0.990 & 1.04 & -1.42 \\
15 & 119 & 2.25 & 0.995 & 1.05 & -4.32 \\
16 & 118 & 3.24 & 0.993 & 1.04 & -2.13 \\
17 & 112 & 1.70 & 0.990 & 1.01 & -3.90 \\
18 & 118 & 1.53 & 0.995 & 1.03 & -3.12 \\
19 & 155 & 2.14 & 0.988 & 0.97 & -1.28 \\
20 & 120 & 2.98 & 0.994 & 0.99 & -4.49 \\
\hline
\end{tabular}

Table reports the number of samples $(n)$ in each trial, the root-meansquare difference (RMS), the correlation coefficient $(r)$, and the bestfit-line coefficients $m$ and $b$

95\% confidence interval (with few exceptions) and possess a mean difference of only $0.99^{\circ}$. In keeping, the correlation plot for this same example trial, Fig. 7b, reveals tight

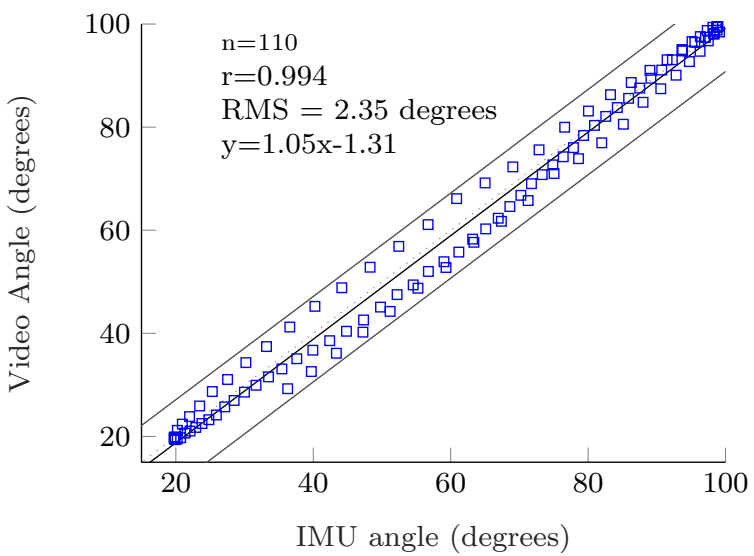

(b)

lines illustrate the $95 \% \mathrm{CI}$ of the agreement limits $(-5.6,7.6)$. b Correlation plot with the superimposed best-fit line (solid) and 95\% CI (dashed)

clustering ( $r=0.994)$ about the best fit line with nearly all data within the $95 \%$ confidence interval.

The findings from this example trial are consistent with the results reported in Tables 1 and 2 across all trials. For the Bland-Altman results of Table 1, note that the mean differences and the bias errors for each trial lie within the range of $\left(0.17^{\circ}<\right.$ bias error $\left.<5.71^{\circ}\right)$, and remain less than $5 \%$ of the corresponding ROM of the pole lean angles. It is also noted that the normality test failed in two trials; refer to $p$ values for the KS test for trials 5 and 7. After a visual inspection, no apparent qualitative difference was found between these two trials and those that passed the normality test and a logarithmic transformation was applied to these two trials, as recommended in [28]. For the correlation results of Table 2, note that the two methods remain correlated $(0.982$ $<r<0.997)$ with small root-mean-square differences $\left(1.53^{\circ}\right.$ $<$ rms $<4.51^{\circ}$ ).

The above results clearly demonstrate agreement between the lean angles estimated by both measurement modalities. The remaining differences are likely unimportant for the stated goal of using the embedded IMU as the means to understand poling dynamics during skiing on outdoor courses. The pole may bend during the pole plant phase, and thus the angle near the top of the pole (where the IMU is mounted) might differ from the angle at the bottom of the pole. Previous attempts to quantify ski pole bending are based on direct distance measurement systems (such as wires) [31], or indirect measurements using laser or infrared video motion capture systems [10,31]. These methods are confined to laboratory conditions and not suitable for outdoor (e.g., competition-like) conditions which is the focus of this study. 


\subsection{Pole-terrain contact events}

The IMU embedded within the grip yields data sufficient to determine the plant and swing phases of the poling cycle. These phases are separated by the instant when the tip first contacts the terrain (end of swing phase and beginning of plant phase) and the instant when the tip subsequently loses contact with the terrain (end of plant phase and start of swing phase). These critical pole-terrain contact events are detected as described in the Methods, and the results from a sample trial are reported next.

Figure 8 illustrates three important experimental quantities plotted over a representative poling cycle. The black curve denotes the vertical position ( $y$ coordinate) of the lower marker on the pole as measured from the video images. The dotted portion of this curve denotes the plant phase when the tip of the pole remains planted on the terrain as confirmed by video images. The blue curve denotes the magnitude of the pole acceleration sampled at the grip (similar to that illustrated in Fig. 4). The instant when the tip first contacts the terrain occurs at the peak acceleration magnitude (marked by black asterisks). Shortly following this instant, the pole rotates about the planted tip of the pole and the acceleration at the grip satisfies the constraint condition given by Eq. (2). That constraint condition is plotted in green and it remains satisfied, hence the constraint magnitude remains close to zero, until the tip lifts from the terrain when the constraint is no longer satisfied, and the constraint magnitude sharply increases. As illustrated, this sharp increase also arises when the vertical position achieves its minimum and then increases thereafter as the tip lifts from the terrain. Thus, the duration of the pole plant phase, which is responsible for active skier propulsion, lies between these two contact events (i.e., between the pairs of asterisks) and these important events are detectable from the grip-embedded IMU data.

The times of pole plant and lift were estimated in this manner from the IMU data across all 40 poling cycles included in this study. The same times were estimated from the video images. The differences in these times from the two measurement methods were computed across all poling cycles and descriptive statistics are summarized in Table 3, which reports the mean, maximum and minimum differences in these times for both the pole plant and pole lift events. From these distributions, the mean and standard deviation of the differences in pole plant times are $-4 \pm 15 \mathrm{~ms}$, while those for the pole lift times are $-7 \pm 11 \mathrm{~ms}$. Thus, the two measurement methods yield similar times of pole plant and pole lift (within $-7 \mathrm{~ms}$ on average). The remaining differences likely arise from the challenge of identifying the pole-terrain contact events in the video images, some (small) sensitivity to the threshold used in the constraint Eq. (2), and any possible residual sliding of the pole tip while contacting the terrain.

Table 3 Summary of the differences between the instants of pole plant and pole lift as detected from the IMU data and the video images across all 40 poling cycles

\begin{tabular}{llll}
\hline & Mean (ms) (STD) & $\begin{array}{l}\text { Max. differ- } \\
\text { ence }(\mathrm{ms})\end{array}$ & $\begin{array}{l}\text { Min. Dif- } \\
\text { ference } \\
(\mathrm{ms})\end{array}$ \\
\hline Pole plant & $-4(15)$ & 0.029 & -0.031 \\
Pole lift & $-7(11)$ & 0.017 & -0.025 \\
\hline
\end{tabular}

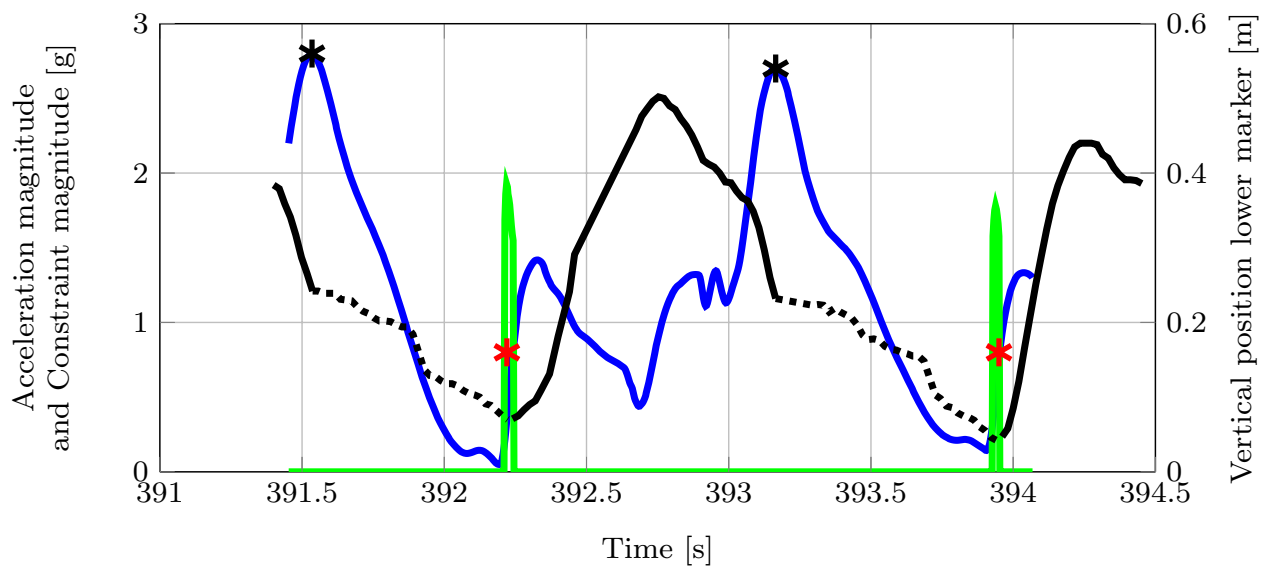

Fig. 8 Detection of pole-terrain contact events. The blue curve represents the magnitude of the acceleration sampled at the grip-embedded IMU. The asterisks denote the pole plant and pole lift instants as determined by the video analysis. The black curve represents the vertical position ( $y$ coordinate) of the lower marker attached to the pole as measured by video images with the dotted portion denoting when the tip of the pole remains planted in the terrain. The green curve represents the constraint condition, Eq. (2). The constraint condition is evaluated when the threshold is exceeded, and the value of the constraint is multiplied by a factor of 50 to aid visualization (color figure online) 


\subsection{Further remarks and future work}

A future research effort could combine this method with a pole-embedded load cell to simultaneously estimate pole force. Combining the force and angle estimates provides the added capability of estimating the horizontal pole ground reaction that propels the skier forward. The resulting technology may further reveal poling performance across athletic populations.

\section{Conclusions}

This paper contributes a new and non-invasive technique to measure the pole lean angle while freely skiing on outdoor courses, thereby surpassing the constraints imposed in laboratory studies. The technique employs an IMU embedded in the grip of the pole and companion algorithms that estimate the instantaneous lean angle of the pole while also identifying the instants of pole plant and pole lift. An experiment (using 20 trials with a total of 40 poling cycles) reveals that the IMU-based estimates of the pole lean angle falls within $95 \%$ confidence intervals and that they remain correlated $(r=0.99)$ with those estimated from video images. Similarly, the IMU-based estimates of the instants of pole plant and pole lift exhibit little difference $(-4 \pm 15 \mathrm{~ms}$ for plant and $-7 \pm 11 \mathrm{~ms}$ for lift) with those estimated from the video images. Consequently, this technology holds considerable promise for measuring and evaluating poling performance during free skiing on outdoor courses.

Acknowledgement Open access funding provided by LUT University.

Open Access This article is licensed under a Creative Commons Attribution 4.0 International License, which permits use, sharing, adaptation, distribution and reproduction in any medium or format, as long as you give appropriate credit to the original author(s) and the source, provide a link to the Creative Commons licence, and indicate if changes were made. The images or other third party material in this article are included in the article's Creative Commons licence, unless indicated otherwise in a credit line to the material. If material is not included in the article's Creative Commons licence and your intended use is not permitted by statutory regulation or exceeds the permitted use, you will need to obtain permission directly from the copyright holder. To view a copy of this licence, visit http://creativecommons.org/licenses/by/4.0/.

\section{References}

1. Nilsson J, Jakobsen V, Tveit P, Eikrehagen O (2003) Pole length and Ground reaction forces during maximal double poling in skiing. Sports Biomech 2(2):227-236

2. Marsland F, Mackintosh C, Holmberg HC, Anson J, Waddington G, Lyons K et al (2017) Full course macro-kinematic analysis of a $10 \mathrm{~km}$ classical cross-country skiing competition. PLoS One 12(8):1-15

3. Millet GY, Hoffman MD, Candau RB, Clifford PS (1998) Poling forces during roller skiing: effects of grade. Med Sci Sports Exerc 30(11):1637-44

4. Holmberg J, Wagenius P (2003) A biomechanical model of a double-poling skier. In: International Society of Biomechanics XIXth Congress: the human body in motion, 6-11 (July 2003) Dunedin New Zealand. Dunedin, NZ

5. Stöggl TL, Holmberg HC (2016) Double-poling biomechanics of elite cross-country skiers: flat versus uphill terrain. Med Sci Sports Exerc 48(8):1580-1589

6. Holmberg HC, Lindinger S, Stöggl T, Eitzlmair E, Müller E (2005) Biomechanical analysis of double poling in elite crosscountry skiers. Med Sci Sports Exerc 37(5):807-818

7. Holmberg LJ, Lund AM (2008) A musculoskeletal full-body simulation of cross-country skiing. Proc Inst Mech Eng Part P J Sports Eng Technol 222(1):11-22

8. Zory R, Vuillerme N, Pellegrini B, Schena F, Rouard A (2009) Effect of fatigue on double pole kinematics in sprint cross-country skiing. Hum Mov Sci 28(1):85-98

9. Forbes SC, Chilibeck PD, Craven B, Bhambhani Y (2010) Comparison of a double poling ergometer and field test for elite cross country sit skiers. N Am J Sports Phys Ther 5(2):40-46

10. Stöggl T, Holmberg HC (2011) Force interaction and 3D pole movement in double poling. Scand J Med Sci Sports 21(6):393-404

11. Nilsson J, Tinmark F, Halvorsen K, Arndt A (2013) Kinematic, kinetic and electromyographic adaptation to speed and resistance in double poling cross country skiing. Eur J Appl Physiol 113(6):1385-1394

12. Pellegrini B, Zoppirolli C, Bortolan L, Zamparo P, Schena F (2014) Gait models and mechanical energy in three cross-country skiing techniques. J Exp Biol 217:3910-3918

13. Smith GA, Nelson RC, Feldman A, Jeffrey L (1989) Analysis of VI skating technique of olympic cross-country skiers. Int J Sport Biomech 5(2):185-208

14. Canclini A, Pozzo R, Moriconi B, Cotelli F (2000) 3D and 2D kinematic analysis of classical technique in elite cross country skiers during a world cup race (S. Caterina 1995) and world championships (Ramsau 1999). Proceedings ICSS—Austria (Ramsau 1999):3-9

15. Federolf P, Reid R, Gilgien M, Haugen P, Smith G (2014) The application of principal component analysis to quantify technique in sports. Scand J Med Sci Sports 24(3):491-499

16. Pellegrini B, Bortolan L, Schena F (2011) Poling force analysis in diagonal stride at different grades in cross country skiers. Scand J Med Sci Sports 21(4):589-597

17. Stöggl T, Karlöf L (2013) Mechanical behaviour of crosscountry ski racing poles during double poling. Sports Biomech 12(4):365-380

18. Myklebust H, Nunez N, Hallén J, Gamboa H (2011) Morphological Analysis of Acceleration Signals in Cross-country Skiing - Information Extraction and Technique Transitions Detection. In: BIOSIGNALS 2011-Proceedings of the International Conference on Bio-inspired Systems and Signal Processing, Rome, Italy, 26-29 January, 2011

19. Myklebust H, Losnegard T, Hallén J (2014) Differences in V1 and V2 ski skating techniques described by accelerometers. Scand J Med Sci Sports 24(6):882-893

20. Marsland F, Lyons K, Anson J, Waddington G, Macintosh C, Chapman D (2012) Identification of cross-country skiing movement patterns using micro-sensors. Sensors 12(4):5047-5066

21. Marsland F, Mackintosh C, Anson J, Lyons K, Waddington G, Chapman DW (2015) Using micro-sensor data to quantify macro 
kinematics of classical cross-country skiing during on-snow training. Sports Biomech 14(4):435-447

22. Fasel B, Spörri J, Schütz P, Lorenzetti S, Aminian K (2017) Validation of functional calibration and strap-down joint drift correction for computing 3D joint angles of knee, hip, and trunk in alpine skiing. PLoS One 12(7):e0181446

23. Ferraris F, Grimaldi U, Parvis M (1995) Procedure for effortless in-field calibration of three-axis rate gyros and accelerometers. Sens Mater 7(5):311-30

24. McGinnis R, Cain S, Davidson S, Vitali R, McLean S, Perkins NC (2014) Validation of complementary filter based IMU data fusion for tracking torso angle and rifle orientation. In: Proceedings of the ASME 2014 international mechanical engineering congress and exposition Montreal, Quebec

25. Fasel B, Favre J, Chardonnens J, Gremion G, Aminian K (2015) An inertial sensor-based system for spatio-temporal analysis in classic cross-country skiing diagonal technique. J Biomech 48(12):3199-3205

26. Bruzzo J (2017) Cross-Country Skiing. Dissertation, Lappeenranta University of Technology
27. Komi PV (1987) Force measurements during cross-country skiing. Int J Sports Biomech 3(4):370-381

28. Bland M, Altman DG (1999) Measuring agreement in method comparison studies. Stat Methods Med Res 8:135-160

29. Massey F (1953) The Kolmogorov-Smirnov test for goodness of Fit. J Am Stat Assoc 46(253):68-78

30. Watson PF, Petrie A (2010) Method agreement analysis: a review of correct methodology. Theriogenology 73:1167-1179

31. Swarén M, Therell M, Eriksson A et al (2013) Testing method for objective evaluation of cross-country ski poles. Sports Eng $16(4): 225-264$

Publisher's Note Springer Nature remains neutral with regard to jurisdictional claims in published maps and institutional affiliations. 